\section{Kidney \\ Blood Pressure Research}

Original Paper
Kidney Blood Press Res 2015;40:266-276

\begin{tabular}{l|l}
\hline DOI: 10.1159/000368502 & $\begin{array}{l}\text { C 2 2015 S. Karger AG, Basel } \\
\text { www.karger.com/kbr }\end{array}$
\end{tabular}

Published online: May 21, 2015

1423-0143/15/0403-0266\$39.50/0

Accepted: April 30, 2015

This is an Open Access article licensed under the terms of the Creative Commons AttributionNonCommercial 3.0 Unported license (CC BY-NC) (www.karger.com/OA-license), applicable to the online version of the article only. Distribution permitted for non-commercial purposes only.

\title{
Sclerostin Quo Vadis? - Is This a Useful Long-Term Mortality Parameter in Prevalent Hemodialysis Patients?
}

\author{
Albina Nowak ${ }^{a}$ Ferruh Artunc ${ }^{b}$ Andreas L. Serrac Emily Pollock ${ }^{a}$

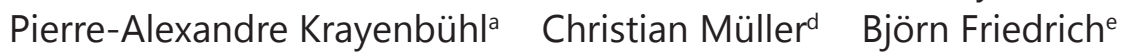 \\ aDepartment of Internal Medicine, University Hospital Zurich, Zurich, Switzerland; 'bepartment of \\ Internal Medicine, Division of Endocrinology, Diabetology, Vascular Disease, Nephrology and Clinical \\ Chemistry, University of Tuebingen, Tuebingen, Germany; ' Epidemiology, Biostatistics, and Prevention \\ Institute (EBPI), University of Zurich, Zurich; dDivision of Cardiology, University Hospital Basel, Basel \\ Switzerland; 'Dialysis Center, Leonberg, Germany
}

\section{Key Words}

Sclerostin • Hemodialysis • Mortality $・$ Cardiovascular

\begin{abstract}
Background/Aims: Cardiovascular calcification contributes to the increased mortality in hemodialysis patients. Sclerostin was identified as an antianabolic bone factor causing soft tissue calcification. Data on prospective large-scale studies associating sclerostin with mortality in hemodialysis patients are so far inconsistent. Methods: In our multicenter prospective longitudinal study following hemodialysis patients, we assessed the associations of sclerostin and bone remodeling markers with long-term mortality. We evaluated the relationship between circulating sclerostin, Fibroblast growth factor 23 (FGF23) and traditional bone remodeling markers. Sclerostin levels in hemodialysis patients were compared with healthy controls. Results: We enrolled 239 hemodialysis patients with a median follow up of 1461 days. In Cox regression analysis, FGF23 (HR 1.40;95\%CI 1.11-1.76), parathyroid hormone (PTH) (HR 1.80;95\%CI 1.44-2.26) and alkaline phosphatase (AP) (HR 1.50;95\%CI 1.10-2.04) per SD, 25(OH)vitamin D (HR 0.42;95\%CI 0.23-0.76) per natural log but not sclerostin (HR 1.02;95\%CI $0.75-1.38$ ) per SD increase were associated with mortality. FGF23, PTH and AP were negatively associated with sclerostin. Among control and hemodialysis females, sclerostin levels were lower than in men. Conclusion: Higher FGF23, PTH, AP and lower 25(OH)vitamin D but not sclerostin predict long-term mortality. Sclerostin was negatively associated with FGF23, PTH and AP and lower in females than in males.
\end{abstract}




\section{Kidney Blood Pressure Research}

Kidney Blood Press Res 2015;40:266-276

\begin{tabular}{l|l}
\hline DOI: 10.1159/000368502 & (c) 2015 S. Karger AG, Basel
\end{tabular}

Published online: May 21, 2015

www.karger.com/kbr

\section{Introduction}

Chronic kidney disease (CKD) is associated with high rates of cardiovascular morbidity and mortality [1]. Renal osteodystrophy and deranged phosphate and calcium homeostasis, accompanied by elevated Fibroblast growth factor 23 (FGF23), parathyroid hormone (PTH) and vitamin D deficiency, have been recognized as crucial cardiovascular risk factors and constitute CKD- mineral and bone disorder (CKD-MBD) syndrome [2]. The simultaneous accelerated calcification of soft tissue and decalcification of bone are driven by the abnormal function of factors involved in mineral metabolism, placing the bone-vascular axis at the center of cardiovascular disease associated with CKD.

Sclerostin is a recently identified osteocyte-secreted 22-kDa glycoprotein that is encoded by the SOST gene. It inhibits osteoblast proliferation and differentiation promoting their apoptosis via canonical Wnt- signaling inhibition [3]. The clinical importance of sclerostin has been emphasized by inherited disorders with excessive bone formation such as sclerostosis [4]; Van Buchem disease [5], "leontiasis ossea" [6]. Wnt-signaling activation has an anabolic and anticatabolic bone effect $[3,4]$. Recent experimental and clinical studies suggest that the Wnt pathway may also play a role in atherosclerosis and vascular calcification: sclerostin was found in atherosclerotic plaques [7-10] and shown to be expressed in calcifying vasculature, which indicates an involvement in transdifferentiation from vascular smooth muscle towards osteocyte-like cells [7]. Data on prospective studies associating sclerostin with cardiovascular calcification and mortality in hemodialysis patients are so far inconsistent.

The aim of this study was to explore all-cause and cardiovascular mortality in a large well-defined prospective hemodialysis patient cohort. Moreover, we aimed to establish a relationship between sclerostin, FGF23 and other bone remodeling factors governing CKDMBD.

\section{Materials and Methods}

This study was approved by the ethics committee of medical faculty Eberhard-Karls-university Germany (project 191/2009B02) and conducted in accordance with the Helsinki declaration. All study participants provided a written informed consent.

\section{Patients}

Patients were eligible if they had given written informed consent, initiation of hemodialysis was more than three months previously and there was no evidence of cardiac event, cardiac amyloidosis or cardiac procedure within the previous two months, no acute life-threatening illness such as malignancy, stroke, frailty, with life expectancy below 6 months, as estimated by the primary treating physician blinded to sclerostin levels. Mortality was prospectively recorded and coded. Patients received status "survived" if they continued attending their regular thrice-weekly hemodialysis facility on 28.08.2014. No patient recovered kidney function. To ascertain the survival status of patients who moved to another dialysis center, the new primary physician was contacted by telephone. The follow-up of 16 transplanted patients was censored at their transplantation date. The cause of death was reported by the primary treating physicians, blinded to the biomarker results and according to their best knowledge. Cardiovascular death was defined as sudden cardiac death, fatal myocardial infarction or fatal ischemic event secondary to cerebrovascular or peripheral vascular disease.

\section{Control group}

The control group consisted of 55 healthy blood donors (37 females and 18 males) who permitted use of their samples for research purposes (age $47 \pm 21$ years). Blinded to sclerostin, we generated ageadjusted groups by excluding hemodialysis patients older than 60 years. The age-adjusted groups included 55 hemodialysis patients aged $48 \pm 10$ years and the 55 controls. 


\section{Kidney \\ Blood Pressure Research}

Patients

the plasma samples were stored at $-80^{\circ} \mathrm{C}$ for further batched analysis when sclerostin, FGF23 and Klotho levels were measured in a blinded fashion in the simultaneously drawn samples. Plasma sclerostin was measured by ELISA (TECOmedical; Sissach, Switzerland) according to the manufacturer's instructions. The limit of detection is $0.008 \mathrm{ng} / \mathrm{ml}$; the lower and upper limits of quantification are 0.01 and $3.34 \mathrm{ng} /$ $\mathrm{ml}$ respectively. Dilution experiments with the assay buffer $(1: 2,1: 4,1: 8)$ showed a mean recovery of 102 $\%$ with a variation coefficient of $6.8 \%$. Plasma FGF23 levels were measured using the two-site second generation Human FGF23 (C-Term) ELISA Kit (Immutopics, San Clemente, CA), soluble $\alpha$-Klotho using ELISA Kit (Immuno-Biologic Laboratories Co., Ltd. Japan), both as previously reported [11].

In a small proportion of patients with low sample volume, we were able to measure either FGF23 or sclerostin. Levels of PTH, AP, serum calcium and phosphate were extracted from the patient medical records and averaged from the available values of the previous year (4-12 values). 25(OH)vitamin D levels were measured 3 months around study enrolment.

\section{Clinical data}

All clinical evaluation of the patients was performed at baseline. Data on residual diuresis ( $24 \mathrm{~h}$ urine in milliliters per day), single pool Kt/V (mean of the last 4 values), interdialytic weight gain, predialytic blood pressure (means from the last 12 values), dialysis access and membrane, dialysis duration, blood pump, arterio-venous fistula flow (measured with a Transonic system, Ithaca, USA) was collected. Body Mass Index (BMI) was calculated as postdialytic dry weight at study enrolment in kilograms divided by the square of the height in meters.

\section{Statistical analysis}

We used descriptive statistics for baseline characteristics and laboratory parameters according to the baseline sclerostin tertiles. Categorical variables were expressed as proportions, continuous variables as means with standard deviations or medians with interquartile ranges [IQR]. Normal distribution was assessed by Kolmogorov-Smirnov-Test. Comparisons of groups were made using one-way analysis of variance (ANOVA), chi-squared, Mann-Whitney and Student test as appropriate. Cox regression analysis was used to examine the mortality risk associated with baseline sclerostin, FGF23, PTH, AP, serum calcium and phosphate levels. Multivariable models were applied to adjust for potential confounders, using prior knowledge of variables that have been associated with mortality risk in hemodialysis patients. We hierarchically adjusted for demographics in model 1 , dialysis-specific risk factors and comorbid conditions in model 2, and albumin, hemoglobin, C-reactive protein and cholesterol in model 3.

In order to separate cardiovascular mortality risk, follow-up of the patients who died from noncardiovascular causes was censored at the day of their death, taking into account that patients dying from non-cardiovascular causes were no longer at risk of dying from cardiovascular causes. Kaplan-Meier analysis was performed and log-rank test of survival distributions equality for the sclerostin tertiles was calculated.

We used logistic regression analysis to explore the association between baseline sclerostin and other bone remodeling markers. The model determination was done using correlation analysis to identify possible collinearity between outcome variable and predictors and subsequently the regression equation was formed by selecting predictors using a prior knowledge of variables that have been associated with sclerostin or possibly be associated according to our opinion. We hierarchically adjusted for demographics in model 1 and dialysis-specific factors, cardiovascular risk factors, mineral metabolism drugs in model 2.

The statistical analyses were performed using the SPSS/PC (version 20.0; SPSS Inc., Chicago, IL, USA) software package. All statistical tests were two-sided, and P values $<0.05$ were considered significant.

\section{Results}

Baseline characteristics and outcome

We enrolled prevalent bicarbonate hemodialysis patients from four centers in Southwest Germany participating in a multicenter prospective study. This cohort was established between September 2009 and April 2010 to investigate the associations between novel biochemical risk parameters and mortality [12-14]. From a total of 250 eligible patients, 239 $(96 \%)$ were enrolled. The exclusion reasons were: six declined to participate, two died within 


\section{Kidney \\ Blood Pressure Research}

Kidney Blood Press Res 2015;40:266-276

DOI: $10.1159 / 000368502$

Published online: May 21, 2015

C 2015 S. Karger AG, Base

www.karger.com/kbr

Patients

Table 1. Baseline characteristics and laboratory parameters according to sclerostin tertiles

\begin{tabular}{|c|c|c|c|c|c|}
\hline & \multirow{2}{*}{$\begin{array}{c}\text { Full cohort } \\
(1.25 \pm 0.46 \mathrm{ng} / \mathrm{ml})\end{array}$} & \multirow{2}{*}{$\begin{array}{c}\begin{array}{c}\text { Sclerostin } \\
\text { Tertile }{ }^{1}\end{array} \\
(<1.01 \mathrm{ng} / \mathrm{ml})\end{array}$} & \multirow{3}{*}{$\begin{array}{c}\text { Sclerostin } \\
\text { Tertile } 2 \\
(1.01-1.37 \mathrm{ng} / \mathrm{ml}) \\
\end{array}$} & \multirow{3}{*}{$\begin{array}{c}\begin{array}{c}\text { Sclerostin } \\
\text { Tertile }^{3}\end{array} \\
(>1.37 \mathrm{ng} / \mathrm{ml}) \\
\mathrm{N}=74\end{array}$} & \multirow[t]{3}{*}{$P$ value } \\
\hline & & & & & \\
\hline & $\mathrm{N}=239$ & $\mathrm{~N}=71$ & & & \\
\hline Age (yr) & $68 \pm 14$ & $67 \pm 15$ & $67 \pm 15$ & $69 \pm 13$ & 0.72 \\
\hline Male gender $\mathrm{n}(\%)$ & $153(64)$ & $30(42)$ & $48(69)$ & $55(74)$ & $<0.001$ \\
\hline Body-mass index * & $26[23-30]$ & $25[22-30]$ & $26[24-30]$ & $27[23-28]$ & 0.40 \\
\hline \multicolumn{6}{|l|}{ Blood pressure (mmHg) } \\
\hline Systolic & 134 [122-144] & $133[121-140]$ & 137 [125-149] & 132 [118-144] & 0.20 \\
\hline Diastolic & $69[63-74]$ & $69[64-73]$ & $71[63-76]$ & $66[62-74]$ & 0.30 \\
\hline \multicolumn{6}{|l|}{ Cause of renal failure n (\%) } \\
\hline Diabetes mellitus & $63(26)$ & $17(24)$ & $24(34)$ & $19(26)$ & 0.30 \\
\hline Hypertension & $19(8)$ & $6(8)$ & $4(6)$ & $5(7)$ & 0.80 \\
\hline Glomerulonephritis & $71(30)$ & $16(23)$ & $22(31)$ & $26(35)$ & 0.20 \\
\hline PKD & $11(4)$ & $4(6)$ & $2(3)$ & $4(5)$ & 0.65 \\
\hline Others / unknown & $75(31)$ & $28(39)$ & $18(26)$ & $20(27)$ & 0.11 \\
\hline \multicolumn{6}{|l|}{ Cardiac comorbidities n (\%) } \\
\hline Coronary artery disease & $73(31)$ & $23(32)$ & $18(26)$ & $22(30)$ & 0.84 \\
\hline Valvular heart disease & $61(26)$ & $15(21)$ & $16(23)$ & $21(28)$ & 0.62 \\
\hline Atrial fibrillation & $54(23)$ & 19 (27) & $15(21)$ & $12(16)$ & 0.35 \\
\hline $\mathrm{LVH}^{\dagger}$ & $101(42)$ & $27(38)$ & $34(49)$ & $32(43)$ & 0.73 \\
\hline \multicolumn{6}{|l|}{ Other comorbidities n (\%) } \\
\hline Diabetes mellitus & $90(38)$ & $24(34)$ & $32(46)$ & $27(36)$ & 0.30 \\
\hline PVD & $80(33)$ & $21(30)$ & $21(30)$ & $25(34)$ & 0.93 \\
\hline Stroke & $38(16)$ & $17(24)$ & $6(9)$ & $12(16)$ & 0.04 \\
\hline Vasculitis & $8(3)$ & $2(3)$ & $4(6)$ & $1(1)$ & 0.36 \\
\hline Malignoma & $34(14)$ & $9(13)$ & $13(19)$ & $10(14)$ & 0.49 \\
\hline \multicolumn{6}{|l|}{ Dialysis specific factors } \\
\hline Dialysis vintage (months) & $59 \pm 53$ & $52 \pm 55$ & $57 \pm 53$ & $67 \pm 53$ & 0.19 \\
\hline Dialysis session (hours) & $4.2 \pm 0.4$ & $4.1 \pm 0.4$ & $4.2 \pm 0.5$ & $4.2 \pm 0.4$ & 0.07 \\
\hline Diuresis (ml/24hours) & $565 \pm 725$ & $528 \pm 703$ & $681 \pm 805$ & $497 \pm 716$ & 0.30 \\
\hline Anuric patients n (\%) & $93(39)$ & $32(45)$ & $25(36)$ & $30(41)$ & 0.44 \\
\hline Singe pool Kt/V & $1.6[1.4-1.7]$ & $1.6[1.3-1.8]$ & $1.5[1.3-1.7]$ & $1.5[1.4-1.7]$ & 0.76 \\
\hline Weight gain $(\mathrm{kg})$ & $1.9[1.3-2.5]$ & $1.7[1.0-2.3]$ & $1.9[1.3-2.7]$ & $2.1[1.4-2.6]$ & 0.11 \\
\hline \multicolumn{6}{|l|}{ Dialysis access at baseline } \\
\hline Arteriovenous fistula n (\%) & $169(71)$ & $42(59)$ & $53(76)$ & $52(70)$ & 0.11 \\
\hline PTFE graft n ( \%) & $31(13)$ & $9(13)$ & $7(10)$ & $7(9)$ & 0.23 \\
\hline Tunnelled catheter n (\%) & $38(16)$ & $19(27)$ & $10(14)$ & $8(11)$ & 0.03 \\
\hline \multicolumn{6}{|l|}{ Dialysis membrane } \\
\hline High-flux n (\%) & $228(95)$ & $68(96)$ & $65(93)$ & $71(96)$ & 0.69 \\
\hline Low-flux n (\%) & $11(5)$ & $3(4)$ & $5(7)$ & $3(4)$ & 0.69 \\
\hline Shunt flow (ml/min) & $565 \pm 725$ & $529 \pm 703$ & $681 \pm 805$ & $497 \pm 716$ & 0.30 \\
\hline Calcium dialysate & $1.4 \pm 0.2$ & $1.4 \pm 0.2$ & $1.4 \pm 0.2$ & $1.4 \pm 0.2$ & 0.29 \\
\hline \multicolumn{6}{|l|}{ Blood results } \\
\hline FGF23 (RU/ml) & $883 \pm 1940$ & $1211 \pm 2963$ & $940 \pm 1592$ & $455 \pm 644$ & 0.08 \\
\hline $\mathrm{PTH}(\mathrm{pg} / \mathrm{ml})$ & $249 \pm 177$ & $311 \pm 228$ & $254 \pm 149$ & $196 \pm 124$ & 0.001 \\
\hline $\mathrm{AP}(\mathrm{U} / \mathrm{l})$ & $91 \pm 42$ & $107 \pm 54$ & $83 \pm 30$ & $85 \pm 39$ & 0.001 \\
\hline Calcium mmol/l & $2.3[2.2-2.4]$ & $2.3[2.2-2.4]$ & $2.3[2.2-2.4]$ & $2.3[2.2-2.4]$ & 0.07 \\
\hline Phosphate mmol/l & $1.6[1.4-1.9]$ & $1.6[1.4-1.9]$ & $1.6[1.4-1.9]$ & $1.6[1.3-1.8]$ & 0.46 \\
\hline C-reactive protein (mg/l) & $12 \pm 12$ & $14 \pm 12$ & $12 \pm 14$ & $11 \pm 10$ & 0.25 \\
\hline $25(\mathrm{OH})$ vitamin D (ng/ml) & 28 [21-35] & 28 [21-35] & 28 [19-34] & $28[23-37]$ & 0.22 \\
\hline Klotho (pg/ml) & $353 \pm 138$ & $374 \pm 122$ & $324 \pm 133$ & $368 \pm 141$ & 0.03 \\
\hline \multicolumn{6}{|l|}{ Medication use n (\%) } \\
\hline Phosphate binders & $205(86)$ & $58(82)$ & $62(89)$ & $63(85)$ & 0.60 \\
\hline Vitamin D replacement & $233(97)$ & $68(96)$ & $68(97)$ & 73 (99) & 0.76 \\
\hline ACE-I or ARB & $126(53)$ & $38(54)$ & $39(56)$ & $38(51)$ & 0.87 \\
\hline Statin & $101(42)$ & 28 (39) & $33(47)$ & $26(35)$ & 0.20 \\
\hline
\end{tabular}

ACE-I, angiotensin converting enzyme inhibitors; AP, alkaline phosphatase; ARB, angiotensin receptor blockers; FGF23, fibroblast growth factor 23; LVH, left ventricular hypertrophy; PKD, polycystic kidney disease; PTH, parathyroid hormone; PVD, peripheral vascular disease. 


\section{Kidney Blood Pressure Research}

\section{Kidney Blood Press Res 2015;40:266-276}

DOI: $10.1159 / 000368502$

Published online: May 21, 2015

(C) 2015 S. Karger AG, Basel

www.karger.com/kbr

Nowak/Artunc/Serra/Pollock/Krayenbühl/Müller/Friedrich: Sclerostin in Hemodialysis Patients

Table 2. Hazard Ratios (and 95\% CIs) for death of novel and traditional bone remodeling marker levels

\begin{tabular}{|c|c|c|c|c|c|c|c|c|}
\hline \multirow[b]{2}{*}{ Variable } & \multicolumn{2}{|c|}{ Unadjusted } & \multicolumn{2}{|c|}{ Adjusted Model $1 *$} & \multicolumn{2}{|c|}{ Adjusted Model $2 \dagger$} & \multicolumn{2}{|c|}{ Adjusted Model 3 ‡ } \\
\hline & $\begin{array}{c}\text { HR } \\
(95 \% \mathrm{CI})\end{array}$ & P value & $\begin{array}{c}\text { HR } \\
(95 \% \mathrm{CI})\end{array}$ & P value & $\begin{array}{c}\mathrm{HR} \\
(95 \% \mathrm{CI}) \\
\end{array}$ & P value & $\begin{array}{c}\text { HR } \\
(95 \% \mathrm{CI})\end{array}$ & P value \\
\hline Sclerostin 1 & $\begin{array}{c}0.92 \\
(0.74-1.16)\end{array}$ & 0.48 & $\begin{array}{c}0.86 \\
(0.67-1.12)\end{array}$ & 0.26 & $\begin{array}{c}1.02 \\
(0.76-1.38)\end{array}$ & 0.88 & $\begin{array}{c}1.02 \\
(0.75-1.38)\end{array}$ & 0.91 \\
\hline FGF23 1 & $\begin{array}{c}1.29 \\
(1.07-1.56)\end{array}$ & 0.008 & $\begin{array}{c}1.40 \\
(1.15-1.71)\end{array}$ & 0.001 & $\begin{array}{c}1.44 \\
(1.13-1.83)\end{array}$ & 0.003 & $\begin{array}{c}1.40 \\
(1.11-1.76)\end{array}$ & 0.004 \\
\hline PTH $^{1}$ & $\begin{array}{c}1.43 \\
(1.19-1.71)\end{array}$ & $<0.001$ & $\begin{array}{c}1.49 \\
(1.22-1.80)\end{array}$ & $<0.001$ & $\begin{array}{c}1.65 \\
(1.32-2.06)\end{array}$ & $<0.001$ & $\begin{array}{c}1.80 \\
(1.44-2.26)\end{array}$ & $<0.001$ \\
\hline $\mathrm{AP} 1$ & $\begin{array}{c}1.21 \\
(1.02-1.43)\end{array}$ & 0.03 & $\begin{array}{c}1.32 \\
(1.10-1.57)\end{array}$ & 0.002 & $\begin{array}{c}1.36 \\
(1.03-1.78)\end{array}$ & 0.03 & $\begin{array}{c}1.50 \\
(1.10-2.04)\end{array}$ & 0.01 \\
\hline Calcium $^{2}$ & $\begin{array}{c}0.04 \\
(0.002-0.86)\end{array}$ & 0.04 & $\begin{array}{c}0.08 \\
(0.003-1.73)\end{array}$ & 0.11 & $\begin{array}{c}0.13 \\
(0.002-10.62)\end{array}$ & 0.37 & $\begin{array}{c}0.38 \\
(0.004-35.69)\end{array}$ & 0.67 \\
\hline Phosphate ${ }^{1}$ & $\begin{array}{c}0.93 \\
(0.76-1.15)\end{array}$ & 0.52 & $\begin{array}{c}1.06 \\
(0.85-1.31)\end{array}$ & 0.62 & $\begin{array}{c}1.12 \\
(0.87-1.46)\end{array}$ & 0.38 & $\begin{array}{c}1.06 \\
(0.82-1.38)\end{array}$ & 0.65 \\
\hline $25(\mathrm{OH})$ vitamin $\mathrm{D}^{2}$ & $\begin{array}{c}0.85 \\
(0.54-1.35)\end{array}$ & 0.49 & $\begin{array}{c}0.62 \\
(0.38-1.03)\end{array}$ & 0.06 & $\begin{array}{c}0.41 \\
(0.23-0.75)\end{array}$ & 0.003 & $\begin{array}{c}0.42 \\
(0.23-0.76)\end{array}$ & 0.004 \\
\hline
\end{tabular}

${ }^{1}$ per $\mathrm{SD} ;{ }^{2}$ per natural log increase.

AP, alkaline phosphatase; CI, confidence interval; FGF23, fibroblast growth factor 23; PTH, parathyroid hormone.

* Model 1=demographics: adjusted for age, gender (male) and by dialysis center clustering.

+ Model 2 = dialysis specific risk factors and comorbid conditions: adjusted for covariates in Model 1 plus dialysis vintage, systolic and diastolic blood pressure, body-mass index, vascular access on study enrolment (fistula, graft, catheter), coexisting conditions listed in Table 1 (coronary artery disease, valvular heart disease, atrial fibrillation, pulmonary hypertension, implantable cardioverter defibrillator carrier; diabetes mellitus, peripheral vascular disease, stroke, vasculitis, malignoma,) cause of renal failure (diabetic nephropathy, hypertensive nephropathy, glomerulonephritis, polycystic kidney disease, others / unknown), medication use listed in Table 1 (phosphate binders, vitamin D replacement, angiotensin converting enzyme inhibitors or angiotensin receptor blockers, beta blockers, aspirin, anticoagulant or clopidogrel, statin), pooled Kt/V.

₹ Model 3 =fully adjusted model: adjusted for covariates in Model 2 plus albumin, hemoglobin, C-reactive protein, cholesterol.

two weeks after enrolment, two suffered from cardiac amyloidosis and one had initiated hemodialysis less than three months before. One patient was lost to follow up. Ninety patients (38\%) died within the median follow-up of 1461 [735-1517] days: 18 (20\%) of a sudden cardiac death, $17(19 \%)$ of an ischemic cardiovascular event, 13 (14\%) of an infection, 6 (7\%) of a malignancy, $25(28 \%)$ of dialysis stop due to frailty, in $11(12 \%)$ the cause of death was unknown. Sclerostin at enrolment was measured in 80 (89\%) non-survivors and 141 (95\%) survivors, FGF23 in 87 (97\%) non-survivors and 147 (99\%) survivors. The baseline characteristics and laboratory results are given in Table 1 . Sclerostin levels were correlated with hemodialysis vintage $(\mathrm{R}=0.18 ; \mathrm{P}=0.006)$ and $\mathrm{CRP}(\mathrm{R}=0.15 ; \mathrm{P}=0.03)$. There was no correlation with age, BMI, KT/V, residual diuresis and sKlotho (data not shown).

\section{Mortality}

Mean sclerostin $(1.21 \pm 0.43$ vs $1.27 \pm 0.48 \mathrm{ng} / \mathrm{ml}, \mathrm{P}=0.38)$ and phosphate $(1.62 \pm 0.37$ vs $1.65 \pm 0.40 \mathrm{mmol} / \mathrm{l}, \mathrm{P}=0.62)$ as well as median $25(\mathrm{OH})$ vitamin $\mathrm{D}(27$ [19-34] vs 28 [22-35] $\mathrm{ng} / \mathrm{ml}, \mathrm{P}=0.62$ ) levels were similar in non-survivors and survivors. Mean FGF23 levels tended to be higher $(1170 \pm 2582$ vs $712 \pm 1413 \mathrm{RU} / \mathrm{ml}, \mathrm{P}=0.08)$, mean PTH $(299 \pm 205$ vs $223 \pm 152$ $\mathrm{pg} / \mathrm{ml}, \mathrm{P}=0.001)$ and AP (98 \pm 46 vs $86 \pm 39 \mathrm{U} / \mathrm{l}, \mathrm{P}=0.03$ ) levels were higher in non-survivors than in survivors. Median calcium levels tended to be lower in non-survivors than in survivors (2.20 [2.18-2.38] vs 2.30 [2.22-2.41] mmol/l, $\mathrm{P}=0.06)$. In Cox regression analysis, sclerostin, phosphate and $25(\mathrm{OH})$ vitamin D levels were not associated with all-cause mortality in an unadjusted model. In contrast, higher FGF23, PTH, AP and lower calcium levels were associated with mortality in an unadjusted model. In model 1 and 2, higher FGF23, PTH and AP and lower calcium and 25(OH)vitamin D levels were associated with mortality. In a fully adjusted model, only higher FGF23, PTH and AP and lower 25(OH) vitamin D levels were associated with mortality (Table 2). If analysed for cardiac mortality only, no association for sclerostin in unadjusted (HR 1.17; 95\%CI 0.85-1.63) and adjusted models (HR 1.03; 95\%CI 0.70-1.51, for model 1; HR 1.10; 95\%CI 0.71-1.70, for model 2; HR 1.17; 95\%CI 0.74-1.84, 


\section{Kidney \\ Blood Pressure Research}

for model 3, all per SD increase) was found in Cox regression.

We avoided collinearity of bone remodelling markers into a Cox regression analysis by adding predictors to separate models and by applying univariate analysis. Cumulative survival among the three groups, stratified according to the baseline sclerostin tertiles, was similar in a Kaplan-Meier analysis $(\mathrm{P}=0.94)$ (Figure 1).

Association of sclerostin with other bone remodelling parameters at baseline

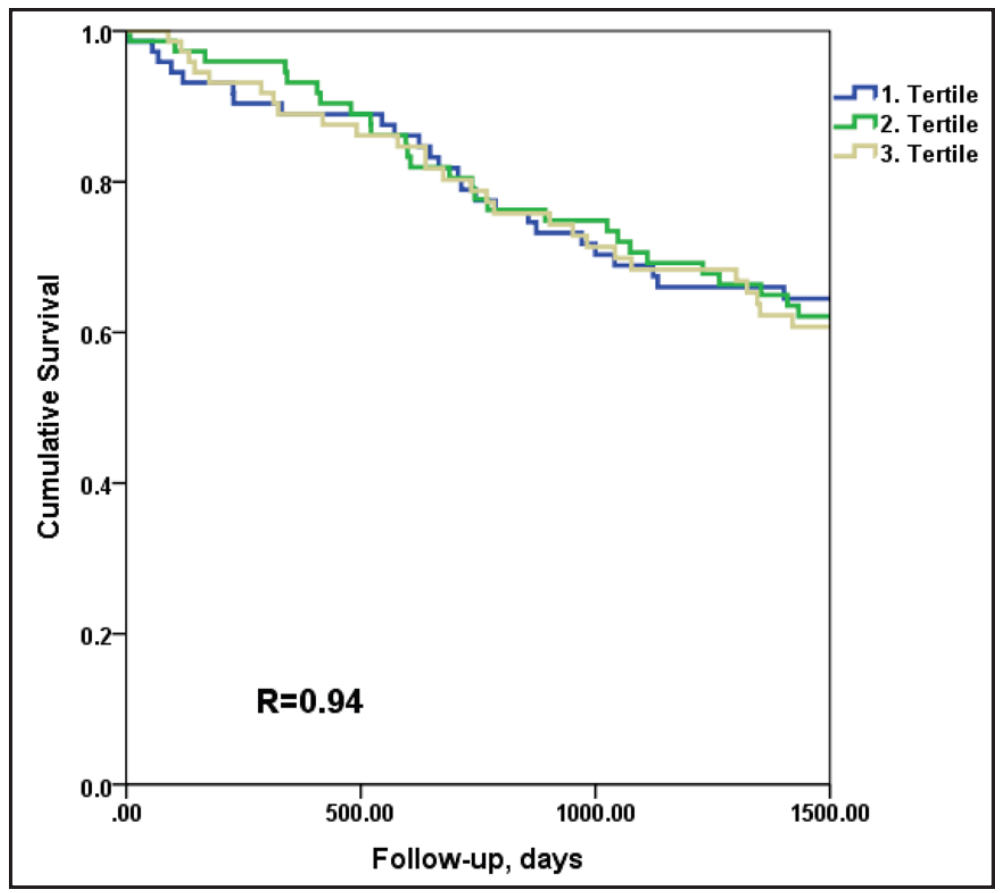

Sclerostin negative-

Fig. 1. Cumulative survival by sclerostin tertiles. ly correlated with FGF23, PTH and AP (Table 3). There was no correlation between sclerostin and 25(OH)vitamin D levels ( $\mathrm{R}=0.02, \mathrm{P}=0.19)$.

In unadjusted regression analysis, FGF23, PTH, AP, phosphate and calcium were determinants of sclerostin levels. In a fully adjusted model, only FGF23, PTH and AP were independent determinants of sclerostin levels (Table 4).

Sclerostin in controls versus hemodialysis patients

The mean sclerostin level in controls was lower than in hemodialysis patients before $(0.58 \pm 0.26$ vs $1.25 \pm 0.46 \mathrm{ng} / \mathrm{ml}, \mathrm{P}<0.001)$ and after age adjustment $(0.58 \pm 0.26$ vs $1.29 \pm$ $0.51 \mathrm{ng} / \mathrm{ml}, \mathrm{P}<0.001)$.

Among females, sclerostin levels were lower in controls than in hemodialysis patients, the same was true among males (Figure 2). Sclerostin levels correlated with age in controls $(\mathrm{R}=0.52, \mathrm{P}<0.001)$, but not in hemodialysis patients $(\mathrm{R}=-0.1, \mathrm{P}=0.86)$.

Table 3. Bivariate correlation of Sclerostin with other bone remodeling markers

\begin{tabular}{|c|c|c|c|c|c|c|}
\hline & Sclerostin & FGF23 & PTH & AP & Calcium & Phosphate \\
\hline FGF23 & $\begin{array}{c}R=-0.17 \\
P=0.01\end{array}$ & & & & & \\
\hline PTH & $\begin{array}{l}R=-0.23 \\
P=0.001\end{array}$ & $\begin{array}{c}\mathrm{R}=0.29 \\
\mathrm{P}<0.001\end{array}$ & & & & \\
\hline AP & $\begin{array}{l}R=-0.22 \\
P=0.001\end{array}$ & $\begin{array}{l}R=0.05 \\
P=0.46\end{array}$ & $\begin{array}{c}R=0.19 \\
P=0.004\end{array}$ & & & \\
\hline Calcium & $\begin{array}{l}R=0.13 \\
P=0.06\end{array}$ & $\begin{array}{l}R=0.12 \\
P=0.07\end{array}$ & $\begin{array}{c}R=-0.07 \\
P=0.28\end{array}$ & $\begin{array}{c}R=-0.04 \\
P=0.51\end{array}$ & & \\
\hline Phosphate & $\begin{array}{c}R=-0.12 \\
P=0.08\end{array}$ & $\begin{array}{c}\mathrm{R}=0.48 \\
\mathrm{P}<0.001\end{array}$ & $\begin{array}{c}R=0.17 \\
P=0.008\end{array}$ & $\begin{array}{c}R=0.005 \\
P=0.94\end{array}$ & $\begin{array}{c}R=-0.89 \\
P=0.17\end{array}$ & \\
\hline $25(\mathrm{OH})$ vit $\mathrm{D}$ & $\begin{array}{l}R=0.02 \\
P=0.19\end{array}$ & $\begin{aligned} R & =-0.01 \\
P & =0.94\end{aligned}$ & $\begin{array}{c}R=-0.09 \\
P=0.20\end{array}$ & $\begin{array}{l}R=0.07 \\
P=0.27\end{array}$ & $\begin{array}{c}R=-0.12 \\
P=0.06\end{array}$ & $\begin{array}{l}R=-0.1 \\
P=0.14\end{array}$ \\
\hline
\end{tabular}

AP, alkaline phosphatase; FGF23, fibroblast growth factor 23; PTH, parathyroid hormone. 


\section{Kidney \\ Blood Pressure Research}

Table 4. Univariate and multivariate linear regression analyses between sclerostin as dependent variable and other bone remodeling markers

\begin{tabular}{|c|c|c|c|c|c|c|}
\hline \multirow[b]{2}{*}{ Variable } & \multicolumn{2}{|c|}{ Unadjusted } & \multicolumn{2}{|c|}{ Adjusted Model $1 *$} & \multicolumn{2}{|c|}{ Adjusted Model $2 \dagger$} \\
\hline & $\beta(95 \% \mathrm{CI})$ & P value & $\beta(95 \% \mathrm{CI})$ & P value & $\beta(95 \% \mathrm{CI})$ & P value \\
\hline FGF23 1 & $0.32(0.23-0.54)$ & $<0.001$ & $-0.03(-0.1$ to 0.03$)$ & 0.27 & $-0.06(-0.14$ to -0.02$)$ & 0.01 \\
\hline $\mathrm{PTH}^{1}$ & $0.71(0.48-0.62)$ & $<0.001$ & $-0.12(-0.15$ to -0.03$)$ & 0.004 & $-0.17(-0.19$ to -0.08$)$ & $<0.001$ \\
\hline $\mathrm{AP}^{1}$ & $0.82(0.41-0.49)$ & $<0.001$ & $-0.09(-0.12$ to -0.03$)$ & 0.26 & $-0.15(-0.14$ to -0.03$)$ & 0.004 \\
\hline Calcium $^{2}$ & $0.19(0.13-0.25)$ & $<0.001$ & $0.08(-0.22$ to 0.38$)$ & 0.57 & $0.15(-0.37$ to 0.67$)$ & 0.56 \\
\hline Phosphate $^{1}$ & $0.9(0.26-0.30)$ & $<0.001$ & $0.18(0.01-0.12)$ & 0.02 & $-0.12(-0.10$ to 0.03$)$ & 0.26 \\
\hline $25(\mathrm{OH})$ vitamin $\mathrm{D}^{2}$ & $0.93(0.36-0.40)$ & $<0.001$ & $0.58(0.13-0.35)$ & $<0.001$ & $0.20(-0.05$ to 0.21$)$ & 0.23 \\
\hline
\end{tabular}

$25(\mathrm{OH})$ vitamin $\mathrm{D}^{2} \quad 0.93(0.36-0.40)$

${ }^{1}$ per SD; ${ }^{2}$ per natural log increase.

AP, alkaline phosphatase; CI, confidence interval; FGF23, fibroblast growth factor 23; PTH, parathyroid hormone.

* Model 1= adjusted for age, gender (male) and dialysis center clustering;

† Model 2 = Model 1 plus dialysis vintage, body-mass index, Diabetes mellitus, Phosphate binders, Vitamin D replacement, pooled KT/V.

\section{Discussion}

In this study, we observed three major phenomena. Firstly, higher FGF23, PTH, AP and lower $25(\mathrm{OH})$ vitamin $\mathrm{D}$ but not sclerostin levels were associated with longterm all-cause mortality after adjustment for potential confounders. An analysis for cardiovascular mortality alone did not improve the association with sclerostin. Secondly, sclerostin levels were negatively correlated with FGF23, PTH and AP; in a fully adjusted model, higher FGF23, PTH and AP were independent determinants of lower sclerostin levels. Thirdly, sclerostin levels were lower in female than in male subjects and lower in controls than in hemodialysis patients.

Our data stimulate further discussion about the role of mineral metabolism and accelerated arterial calcification leading to cardiovascular morbidity and mortality in hemodialysis patients. Previous studies examining sclerostin as a predictor for adverse outcomes have in certain cases delivered inhomogeneous results. A study by Viaene et al found a trend towards survival benefit with higher circulating sclerostin levels in 100 hemodialysis patients after a median follow-up time of 637 days [15], suggesting that sclerostin might be a calcification inhibitor. In contrast, Kanbay et al found higher sclerostin values to be associated with fatal and non-fatal cardiovascular events in 173 non-dialyzed patients with CKD stages 3-5 after a mean follow-up of 26 months [16]. A very recent study by Drechsler et al found high sclerostin levels to be protective for cardiovascular mortality in 673 incident dialysis patients [17]. The findings of Delanaye et al could not support these results - they found no association between sclerostin and mortality after 2 years in a observational study with 164 hemodialysis patients [18]. The mortality rates in studies by Viaene et al and Delanye et al, both examining prevalent hemodialysis patients over a follow-up period of $\sim 2$ years, are slightly higher ( $\sim 15 \%$ per year) as compared to our study ( $\sim 10 \%$ per year). Nevertheless, 


\section{Kidney Blood Pressure Research}

Kidney Blood Press Res 2015;40:266-276

\begin{tabular}{l|l}
\hline DOI: $10.1159 / 000368502$ & (C) 2015 S. Karger AG, Basel
\end{tabular}

Published online: May 21, 2015

www.karger.com/kbr

the finding of no significant correlation between sclerostin levels and all-cause mortality is similar among these both and our studies. The novelty of our study is that we extended the follow-up time to $\sim 4$ years and augmented the observed prevalent cohort to 239 patients.

The potential reasons for these differences remain somewhat unclear. The case-mix of patients was different between studies. In particular, the study by Viaene et al contained a higher proportion of patients with diabetes mellitus and had an overall shorter dialysis vintage in the hemodialysis cohort as compared to our study. Kanbay et al enrolled nondialyzed CKD patients. The well-powered prospective cohort study from the Netherlands (NECOSAD) by Drechsler et al included incident (and not prevalent) patients of younger age as compared to our study, $7 \%$ of them were on peritoneal dialysis. Moreover, the use of phosphate binders and vitamin D supplementation as well as dialysis specific factors like residual diuresis and cardiovascular comorbities are not shown in the study. However, these factors may play a prominent role in influencing mortality and could partly explain the lack of similarity to our results.

Another potential inhomogeneity source is that two different ELISAs for circulating sclerostin were used in the aforementioned studies: TECOmedical in the present and in the study by Drechsler et al and Biomedica in study by Viaene et al and Kanbay et al. Although the assays show a strong correlation, the intra- and inter-precision for TECO $(<10 \%)$ was shown to be smaller than for Biomedica $(<20 \%)$ in another recent study by Costa et al [19].

The cause of the lack of association of circulating sclerostin with mortality shown in this study remains to be elucidated. A possible explanation is that sclerostin accumulates in hemodialysis patients, not only due to its enhanced production in bones and possibly calcifying tissues but also due to its absent or very low elimination due to low residual kidney function. An important role of kidney function in sclerostin clearance was shown in studies by Pelletier et al [20]. Due to its low molecular weight and positive charge [21], sclerostin is filtered across the glomerular membrane and reabsorbed from urine [22]. Also the study by Bonani et al showed a dramatic sclerostin decrease after kidney transplantation in patients with ESRD. This effect can predominantly be explained by the improved kidney function [23].

Interestingly, we found sclerostin levels in hemodialysis patients to be considerably higher than in the control group, which is in line with previous results $[15,16,24]$.This phenomenon could be explained not only by reduced clearance in impaired kidney function, but also by an enhanced production in uremia. Cejka et al were the first to document increased renal sclerostin elimination with declining kidney function suggesting an increased sclerostin production in uremia [22] . In agreement, Sabbagh et al reported an increased expression of the SOST gene and other Wnt antagonists in the bone biopsies of CKD mice [25]. In early CKD, sclerostin and other Wnt pathway inhibitors were shown to induce a mineral and bone disorder in an experimental mouse model $[9,26]$ and in humans [9]. Sclerostin expression was found in the aortas of patients undergoing aortic valve replacement conducted secondary to aortic calcification [27]. Brandenburg et al found an association of sclerostin with calcifying aortic valve disease [24] in 67 chronic hemodialysis patients. An animal study by Zhu et al showed sclerostin to be upregulated in vascular calcification models [7].

Of note, FGF23 and iPTH were negatively correlated with sclerostin levels in our study. Both hormones exert a phosphaturic action, FGF23 increases followed by PTH increase with advancing phosphate retention during the progression of kidney disease [28]. Concordantly, PTH receptor signalling is a known inhibitor of sclerostin expression [29]. PTH was shown to reduce sclerostin expression in osteocytes in culture and animal models [29-31], systemic PTH treatment was followed by a reduction of sclerostin levels in postmenopausal women [32].

In summary, we hypothesize that the negative association of sclerostin with the other CKD-MBD parameters (FGF23, PTH and AP) may indicate a protective role of sclerostin as an inhibitor of vascular calcification.

Several limitations merit consideration. Firstly, the study was not designed to establish the exact cause of death. This limitation is common and may partly be due to the relatively low autopsy rate [33]. Due to this, the cause of death was established according to the best 


\section{Kidney \\ Blood Pressure Research}

Kidney Blood Press Res 2015;40:266-276

\begin{tabular}{l|l}
\hline DOI: $10.1159 / 000368502$ & (C) 2015 S. Karger AG, Basel
\end{tabular}

Published online: May 21, 2015

www.karger.com/kbr

knowledge of the treating physicians. Secondly, we did not define non-fatal MI as an end-point. Non-fatal MI rarely occurs in hemodialysis patients [34] and coronary media calcifications rather than arterial plaques have a greater impact on mortality in dialysis patients $[35,36]$. We also did not record smoking habits at study enrolment. Thirdly, we cannot report on $1,25(\mathrm{OH})$ vitamin D measurements, as this is not routinely measured in our hemodialysis patients. Similarly, we cannot report on bone specific alkaline phosphatase measurements. On the other hand, conventional rather than bone specific alkaline phosphatase has been recommended to be measured for monitoring and management of CKD-MBD [37] and strongly reflects clinical outcomes in hemodialysis patients [38]. Fourthly, sclerostin, FGF23 and Klotho were simultaneously measured at enrolment, whereas levels of routinely used biomarkers were extracted from the patients' notes meaning not exactly the same time points of measurement. Nevertheless, the significant association of the novel and conventional parameters seems to be in accordance with previous works we discussed above. Another limitation is that in a small proportion of patients with low sample volume, we were able to measure either sclerostin or FGF23. Finally, we did not quantify vascular calcification, perform bone biopsy or densitometry and therefore cannot interpret sclerostin and other bone remodelling marker results in the light of such findings. Further studies including a larger number of patients to examine the effect of sclerostin levels on cardiovascular morbidity and mortality are desirable to explain inhomogeneity among studies and better establish the role of circulating sclerostin on soft tissue calcification and bone formation in CKD.

\section{Conclusion}

Higher FGF23, PTH, AP and lower 25(OH)vitamin D but not sclerostin levels predict long-term mortality. Sclerostin may be considered as one of the important players of CKDMBD in hemodialysis patients.

\section{Disclosure Statement}

The authors of this manuscript state that they do not have any conflict of interests and nothing to disclose.

\section{Aknowlegdments}

The sclerostin, FGF23 and Klotho measurements were provided by TECOmedical AG, Gewerbestrasse 104450 Sissach, Switzerland. The funders were blinded to patients' names and all clinical information, had no role in the initiation or design of the study, collection of samples, analysis, interpretation of data, writing of the paper, or the submission for publication. The study and researchers are independent of the funders, TECOmedical AG.

\section{References}

1 Peralta CA, Shlipak MG, Judd S, Cushman M, McClellan W, Zakai NA, Safford MM, Zhang X, Muntner P, Warnock D: Detection of chronic kidney disease with creatinine, cystatin C, and urine albuminto-creatinine ratio and association with progression to end-stage renal disease and mortality. JAMA 2011;305:1545-1552.

2 Moe S, Drueke T, Cunningham J, Goodman W, Martin K, Olgaard K, Ott S, Sprague S, Lameire N, Eknoyan G, Kidney Disease: Improving Global 0: Definition, evaluation, and classification of renal osteodystrophy: a position statement from Kidney Disease: Improving Global Outcomes (KDIGO). Kidney Int 2006;69:19451953. 


\section{Kidney \\ Blood Pressure Research}

3 Ke HZ, Richards WG, Li X, Ominsky MS: Sclerostin and Dickkopf-1 as therapeutic targets in bone diseases. Endocr Rev 2012;33:747-783.

4 Balemans W, Ebeling M, Patel N, Van Hul E, Olson P, Dioszegi M, Lacza C, Wuyts W, Van Den Ende J, Willems P, Paes-Alves AF, Hill S, Bueno M, Ramos FJ, Tacconi P, Dikkers FG, Stratakis C, Lindpaintner K, Vickery B, Foernzler D, Van Hul W: Increased bone density in sclerosteosis is due to the deficiency of a novel secreted protein (SOST). Hum Mol Genet 2001;10:537-543.

5 Loots GG, Kneissel M, Keller H, Baptist M, Chang J, Collette NM, Ovcharenko D, Plajzer-Frick I, Rubin EM: Genomic deletion of a long-range bone enhancer misregulates sclerostin in Van Buchem disease. Genome Res 2005;15:928-935.

6 Kim SJ, Bieganski T, Sohn YB, Kozlowski K, Semenov M, Okamoto N, Kim CH, Ko AR, Ahn GH, Choi YL, Park SW, Ki CS, Kim OH, Nishimura G, Unger S, Superti-Furga A, Jin DK: Identification of signal peptide domain SOST mutations in autosomal dominant craniodiaphyseal dysplasia. Hum Genet 2011;129:497-502.

7 Zhu D, Mackenzie NC, Millan JL, Farquharson C, MacRae VE: The appearance and modulation of osteocyte marker expression during calcification of vascular smooth muscle cells. PLoS One 2011;6:e19595.

8 Claes KJ, Viaene L, Heye S, Meijers B, d'Haese P, Evenepoel P: Sclerostin: Another vascular calcification inhibitor? J Clin Endocrinol Metab 2013;98:3221-3228.

9 Fang Y, Ginsberg C, Sugatani T, Monier-Faugere MC, Malluche H, Hruska KA: Early chronic kidney diseasemineral bone disorder stimulates vascular calcification. Kidney Int 2014;85:142-150.

10 Morales-Santana S, Garcia-Fontana B, Garcia-Martin A, Rozas-Moreno P, Garcia-Salcedo JA, Reyes-Garcia $\mathrm{R}$, Munoz-Torres M: Atherosclerotic disease in type 2 diabetes is associated with an increase in sclerostin levels. Diabetes Care 2013;36:1667-1674.

11 Nowak A, Friedrich B, Artunc F, Serra AL, Breidthardt T, Twerenbold R, Peter M, Mueller C: Prognostic Value and Link to Atrial Fibrillation of Soluble Klotho and FGF23 in Hemodialysis Patients. PLoS One 2014;9:e100688.

12 Artunc F, Mueller C, Breidthardt T, Twerenbold R, Peter A, Thamer C, Weyrich P, Haering HU, Friedrich B: Sensitive troponins--which suits better for hemodialysis patients? Associated factors and prediction of mortality. PLoS One 2012; 7:e47610.

13 Artunc F, Mueller C, Breidthardt T, Twerenbold R, Rettig I, Usta E, Haring HU, Friedrich B: Comparison of the diagnostic performance of three natriuretic peptides in hemodialysis patients: which is the appropriate biomarker? Kidney Blood Press Res 2012;36:172-181.

14 Artunc F, Nowak A, Mueller C, Breidthardt T, Twerenbold R, Wagner R, Peter A, Haering HU, Ebmeyer S, Friedrich B: Plasma concentrations of the vasoactive peptide fragments mid-regional pro-adrenomedullin, C-terminal pro-endothelin 1 and copeptin in hemodialysis patients: associated factors and prediction of mortality. PLoS One 2014;9:e86148.

15 Viaene L, Behets GJ, Claes K, Meijers B, Blocki F, Brandenburg V, Evenepoel P, D'Haese PC: Sclerostin: another bone-related protein related to all-cause mortality in haemodialysis? Nephrol Dial Transplant 2013;28:3024-3030.

16 Kanbay M, Siriopol D, Saglam M, Gulcan Kurt Y, Gok M, Cetinkaya H, Karaman M, Umut Unal H, Oguz Y, Sari S, Eyileten T, Goldsmith D, Vural A, Veisa G, Covic A, Ilker Yilmaz M: Serum sclerostin and adverse outcomes in non-dialyzed chronic kidney disease patients. J Clin Endocrinol Metab 2014, DOI 10.1210/jc.20142042jc20142042.

17 Drechsler C, Evenepoel P, Vervloet MG, Wanner C, Ketteler M, Marx N, Floege J, Dekker FW, Brandenburg VM, for the NSG: High levels of circulating sclerostin are associated with better cardiovascular survival in incident dialysis patients: results from the NECOSAD study. Nephrol Dial Transplant 2014, DOI 10.1093/ ndt/gfu301.

18 Delanaye P, Krzesinski JM, Warling X, Moonen M, Smelten N, Medart L, Bruyere O, Reginster JY, Pottel $\mathrm{H}$, Cavalier E: Clinical and biological determinants of sclerostin plasma concentration in hemodialysis patients. Nephron Clin Pract 2014;128:127-134.

19 Costa AG, Cremers S, Dworakowski E, Lazaretti-Castro M, Bilezikian JP: Comparison of two commercially available ELISAs for circulating sclerostin. Osteoporos Int 2014;25:1547-1554.

20 Pelletier S, Dubourg L, Carlier MC, Hadj-Aissa A, Fouque D: The relation between renal function and serum sclerostin in adult patients with CKD. Clin J Am Soc Nephrol 2013;8:819-823. 


\section{Kidney \\ Blood Pressure Research}

Kidney Blood Press Res 2015;40:266-276

\begin{tabular}{l|l}
\hline DOI: $10.1159 / 000368502$ & (C) 2015 S. Karger AG, Basel
\end{tabular}

Published online: May 21, 2015

www.karger.com/kbr

21 Veverka V, Henry AJ, Slocombe PM, Ventom A, Mulloy B, Muskett FW, Muzylak M, Greenslade K, Moore A, Zhang L, Gong J, Qian X, Paszty C, Taylor RJ, Robinson MK, Carr MD: Characterization of the structural features and interactions of sclerostin: molecular insight into a key regulator of Wht-mediated bone formation. J Biol Chem 2009;284:10890-10900.

22 Cejka D, Marculescu R, Kozakowski N, Plischke M, Reiter T, Gessl A, Haas M: Renal elimination of sclerostin increases with declining kidney function. J Clin Endocrinol Metab 2014;99:248-255.

23 Bonani M, Rodriguez D, Fehr T, Mohebbi N, Brockmann J, Blum M, Graf N, Frey D, Wuthrich RP: Sclerostin blood levels before and after kidney transplantation. Kidney Blood Press Res 2014;39:230-239.

24 Brandenburg VM, Kramann R, Koos R, Kruger T, Schurgers L, Muhlenbruch G, Hubner S, Gladziwa U, Drechsler C, Ketteler M: Relationship between sclerostin and cardiovascular calcification in hemodialysis patients: a cross-sectional study. BMC Nephrol 2013;14:219.

25 Sabbagh Y, Graciolli FG, O'Brien S, Tang W, dos Reis LM, Ryan S, Phillips L, Boulanger J, Song W, Bracken C, Liu S, Ledbetter S, Dechow P, Canziani ME, Carvalho AB, Jorgetti V, Moyses RM, Schiavi SC: Repression of osteocyte Wnt/beta-catenin signaling is an early event in the progression of renal osteodystrophy. J Bone Miner Res 2012;27:1757-1772.

26 Fang Y, Ginsberg C, Seifert M, Agapova O, Sugatani T, Register TC, Freedman BI, Monier-Faugere MC, Malluche H, Hruska KA: CKD-Induced Wingless/Integration1 Inhibitors and Phosphorus Cause the CKDMineral and Bone Disorder. J Am Soc Nephrol 2014;25:1760-1773.

27 Didangelos A, Yin X, Mandal K, Baumert M, Jahangiri M, Mayr M: Proteomics characterization of extracellular space components in the human aorta. Mol Cell Proteomics 2010;9:2048-2062.

28 Isakova T, Wahl P, Vargas GS, Gutierrez OM, Scialla J, Xie H, Appleby D, Nessel L, Bellovich K, Chen J, Hamm L, Gadegbeku C, Horwitz E, Townsend RR, Anderson CA, Lash JP, Hsu CY, Leonard MB, Wolf M: Fibroblast growth factor 23 is elevated before parathyroid hormone and phosphate in chronic kidney disease. Kidney Int 2011;79:1370-1378.

29 O'Brien CA, Plotkin LI, Galli C, Goellner JJ, Gortazar AR, Allen MR, Robling AG, Bouxsein M, Schipani E, Turner CH, Jilka RL, Weinstein RS, Manolagas SC, Bellido T: Control of bone mass and remodeling by PTH receptor signaling in osteocytes. PLoS One 2008;3:e2942.

30 Kulkarni NH, Halladay DL, Miles RR, Gilbert LM, Frolik CA, Galvin RJ, Martin TJ, Gillespie MT, Onyia JE: Effects of parathyroid hormone on Wnt signaling pathway in bone. J Cell Biochem 2005;95:1178-1190.

31 Bellido T, Ali AA, Gubrij I, Plotkin LI, Fu Q, O'Brien CA, Manolagas SC, Jilka RL: Chronic elevation of parathyroid hormone in mice reduces expression of sclerostin by osteocytes: a novel mechanism for hormonal control of osteoblastogenesis. Endocrinology 2005;146:4577-4583.

32 Drake MT, Srinivasan B, Modder UI, Peterson JM, McCready LK, Riggs BL, Dwyer D, Stolina M, Kostenuik P, Khosla S: Effects of parathyroid hormone treatment on circulating sclerostin levels in postmenopausal women. J Clin Endocrinol Metab 2010;95:5056-5062.

33 Isakova T, Xie H, Yang W, Xie D, Anderson AH, Scialla J, Wahl P, Gutierrez OM, Steigerwalt S, He J, Schwartz S, Lo J, Ojo A, Sondheimer J, Hsu CY, Lash J, Leonard M, Kusek JW, Feldman HI, Wolf M, Chronic Renal Insufficiency Cohort Study G: Fibroblast growth factor 23 and risks of mortality and end-stage renal disease in patients with chronic kidney disease. JAMA 2011;305:2432-2439.

34 Etter C, Straub Y, Hersberger M, Raz HR, Kistler T, Kiss D, Wuthrich RP, Gloor HJ, Aerne D, Wahl P, Klaghofer $\mathrm{R}$, Ambuhl PM: Pregnancy-associated plasma protein-A is an independent short-time predictor of mortality in patients on maintenance haemodialysis. Eur Heart J 2010;31:354-359.

35 London GM, Guerin AP, Marchais SJ, Metivier F, Pannier B, Adda H: Arterial media calcification in end-stage renal disease: impact on all-cause and cardiovascular mortality. Nephrol Dial Transplant 2003;18:17311740 .

36 Tong LL, Mehrotra R, Shavelle DM, Budoff M, Adler S: Poor correlation between coronary artery calcification and obstructive coronary artery disease in an end-stage renal disease patient. Hemodial Int 2008;12:16-22.

37 Magnusson P, Sharp CA, Magnusson M, Risteli J, Davie MW, Larsson L: Effect of chronic renal failure on bone turnover and bone alkaline phosphatase isoforms. Kidney Int 2001;60:257-265.

38 Maruyama Y, Taniguchi M, Kazama JJ, Yokoyama K, Hosoya T, Yokoo T, Shigematsu T, Iseki K, Tsubakihara Y: A higher serum alkaline phosphatase is associated with the incidence of hip fracture and mortality among patients receiving hemodialysis in Japan. Nephrol Dial Transplant 2014;29:1532-1538. 